\title{
Three-Dimensional Particle Image Velocimetry Using a Plenoptic Camera
}

\author{
Kyle Lynch* \\ Delft University of Technology, Delft, $N L$
}

\author{
Tim Fahringer ${ }^{\dagger}$ and Brian Thurow T $^{\ddagger}$ \\ Auburn University, Auburn, AL
}

\begin{abstract}
A novel 3-D, 3-C PIV technique is described, based on volume illumination and a plenoptic camera to measure a velocity field. The technique is based on plenoptic photography, which uses a dense microlens array mounted near a camera sensor to sample the spatial and angular distribution of light entering the camera. Various algorithms are then used to reconstruct a volumetric intensity field after the image is taken, and cross-correlation algorithms extract the velocity field from the reconstructed volume. This paper provides an introduction to the concepts of light fields and plenoptic photography, and describes the algorithms used to reconstruct the measurement volume. A comparison is made between the use of a combined computational refocusing and thresholding approach versus a direct tomographic reconstruction approach. This discussion lays the groundwork for a more detailed study of reconstruction accuracy, achieveable particle number density, reconstruction ambiguities (e.g., ghost particles), and other factors in a following study. Additionally, the construction of a prototype camera based on a 16-megapixel interline CCD sensor is described and preliminary experimental renderings are given.
\end{abstract}

\section{Introduction}

Particle image velocimetry (PIV) has become a well-established measurement technique used extensively to resolve planar velocity fields in a variety of flow environments. However, traditional PIV is not capable of capturing the full three-dimensional (3-D), three-component (3-C) velocity field instantaneously, which is important for quantifying the topology and extent of coherent flow structures which pervade most turbulent flows. Moreover, turbulence is inherently 3-D in nature, and a full description requires a measurement of the 3-D velocity field and derivative quantities such as the stress tensor and the vorticity vector.

These limitations have led to a number of efforts being made over the years to develop 3-D, 3-C PIV techniques. Advances such as stereoscopic-PIV ${ }^{1}$ extend traditional PIV to allow 3-C measurements within a 2-D plane, and dual-plane stereoscopic-PIV ${ }^{2}$ applies this technique to two planes which allows the derivative quantities of each dimension and component to be calculated. However, since these techniques only acquire 3-C data within a single plane or two planes, the out-of-plane spatial resolution is much lower than the inplane resolution. For this reason, these techniques are not considered truly three-dimensional. An additional extension of the aforementioned techniques is scanning PIV, ${ }^{3}$ where high-repetition-rate laser and camera systems are used to illuminate and capture images at multiple planes throughout the measurement volume. The advantage of these systems are the intuitive setup and data processing steps; however, even with $\mathrm{kHz}-$ rate lasers the volume scanning time is often large compared to the characteristic timescales of the flow under consideration and prevents the technique from being applied to most practical flows. These systems are often employed in water tunnels (e.g... Zhang et al. ${ }^{4}$ ).

Four techniques that have received attention for their ability to conduct 3-D, 3-C measurements are defocusing PIV,${ }^{5,6}$ holographic PIV ${ }^{7}$ tomographic PIV ${ }^{8}$ and synthetic aperture PIV. ${ }^{9}$ The former is based on the use of specialized apertures near the camera lens or multiple cameras which eliminates the ambiguity in particle depth that occurs when a particle is not located within the focal plane. Computational algorithms use the knowledge of the aperture shape or camera positions to determine the particle position and depth.

${ }^{*} \mathrm{PhD}$ Candidate, Department of Aerospace Engineering.

${ }^{\dagger}$ Research Assistant, Department of Aerospace Engineering.

$\ddagger$ Associate Professor, Department of Aerospace Engineering. Email: thurow@auburn.edu. 
The strength of this technique is the relative simplicity of the equipment required and ease of analysis; however, the particle density is severely limited since the location of individual particles must be resolved. Also, in the case of a single camera system, the use of an aperture greatly reduces the amount of collected light. The combination of these factors typically restricts the application of the technique to water tunnels where particle density can be precisely controlled and relatively large particles can be used.

Holographic PIV is based on the recording of the interference pattern, or hologram, generated by a reference light beam passing through a volume. The volume is then reconstructed by illuminating the hologram with the same reference light beam or a synthetic reference beam. The resulting volume represents the light intensity field which can then be evaluated to determine particle positions or perform cross-correlation. Most holographic PIV setups utilize specialized holographic films enabling the hologram to be densley sampled and a large number of vectors to be generated. At the same time, the use of films is a disadvantage due to the time-consuming reconstruction and wet processing steps required. Some progress has been made recently in digital holographic PIV using CCD sensors and digitized reconstruction algorithms, but these techniques have much lower resolution and yield fewer velocity vectors. Nevertheless, in all cases the optical complexity of holographic PIV precludes the wide adoption of the technique in the near future.

Tomographic PIV has seen rapid development and maturation, and is now offered as a commercially available system. In this technique, three or more high-resolution CCD cameras are used to image a particle field illuminated by a thick laser sheet. Tomographic algorithms are used to reconstruct the volume, after which cross-correlation algorithms are used to determine the particle displacement. This technique has been demonstrated in a variety of flows including turbulent boundary layers, ${ }^{10}$ cylinder wakes, ${ }^{11}$ and shockwave/turbulent boundary layer interactions. ${ }^{12}$ It has also been adapted to $\mathrm{kHz}$ rates using high-speed cameras for aeroacoustic studies (see Violato et al. ${ }^{13}$ ). Tomo-PIV, however, has some rather significant restrictions that limit its use in many situations. These include the relatively thin $(\sim 10 \mathrm{~mm}$ depth) volume over which a measurement can be made, errors in the volume reconstruction process due to the limited number of viewing angles (e.g. the generation of image artifacts known as ghost particles), limited particle number density, complexity of the experimental arrangement, and the expense of the overall system.

Synthetic aperture PIV (SAPIV) is the most recent advance in 3-D PIV techniques, described by Belden et al. ${ }^{9}$ The technique uses a large camera array (eight or more cameras) to capture multiple views of the measurement volume simultaneously. In contrast to Tomo-PIV, the map-shift-average algorithm is used to construct synthetically refocused images from the individual views by projecting each view onto a common focal surface. In the resulting image, particles that lie on the focal surface are sharp and in-focus, whereas particles off of the surface are blurred. By thresholding the refocused images, the 3-D intensity field is compiled and is used as the input to cross-correlation algorithms. The technique suffers from many of the same restrictions as Tomo-PIV, but unfortunately uses an even greater number of cameras.

This paper describes a novel technique for performing 3-D velocimetry using a single camera, based on the concept of plenoptic photography. The use of a single camera eliminates many of the drawbacks facing current 3-D PIV systems. An introduction to plenoptic photography is given to describe the manner in which the camera operates. Following this, various image processing algorithms are described and demonstrated on synthetic and real data. The resulting volumes are used as inputs to a 3-D cross-correlation algorithm to obtain velocity fields. Finally, the construction of a prototype camera is discussed and preliminary data is presented.

\section{A. Concepts of Light Field Photography}

The concept of light field imaging has evolved over the past 17 years beginning with the work of Adelson and Wang, ${ }^{14}$ and revisited by Ng. et al. ${ }^{15}$ for handheld photography and Levoy et al. ${ }^{16}$ for microscopy. These recent works describe the light field as the complete distribution of light rays in space which are described by a 5 -D function, sometimes termed the plenoptic function, where each ray is parameterized by its position $(x, y, z)$ and angle of propagation $(\theta, \phi)$. In a transparent medium such as air, the light ray propagates in approximately a straight line, making one of the spatial coordinates redundant and the resulting 4-D parameterization is commonly termed the light field. Conventional photography only captures the spatial distribution of the 4-D light field because the angular distribution is lost through integration at the sensor surface.

In contrast, a device that can record the complete 4-D light field would be of tremendous value. As described in Levoy, ${ }^{17}$ there are several ways to capture a light field including the mounting of a camera on a gantry and taking a large number of photos at different positions, the use of a large array of cameras, or 
the use of a microlens array mounted near a CCD to encode this information onto a single sensor. This last device, which is termed the plenoptic camera, records the light field on a single image and is the focus of the development in this work.

Our schematic description of the plenoptic camera follows that of Adelson and Wang ${ }^{14}$ who were the first to propose the concept of the plenoptic camera. They used the analogy of a single lens stereo view to describe the function of the camera. Figure 1 illustrates this principle. In figure 1a, a point object is focused onto an image sensor leading to a bright distinct point on the sensor surface. As the object is moved closer (fig. 1b) and farther away (fig. 1c), the image of the spot grows larger and appears blurred as it goes out of focus. At this point, the position of the object is not apparent from the image; however, if an eccentric aperture is used, the distance of the object from the lens can be determined. In figure 2a, when the object is in focus, the light strikes the same point as before and produces a sharp peak at the center of the sensor. When the object is moved closer to the lens (fig. 2b), the eccentric aperture limits the angular range of rays traveling to the sensor, leading to a blurred image of the object that is displaced to the left of center. Conversely, when the object is moved further away, the rays follow a different path through the aperture with the image formed in front of the sensor and the resulting rays traveling to the right of center. Thus, a close-up object leads to lateral displacement on the sensor to the left whereas a distant object leads to displacement to the right. As such, the precise location and depth of the object can be determined by measuring its horizontal displacement and size on the sensor.
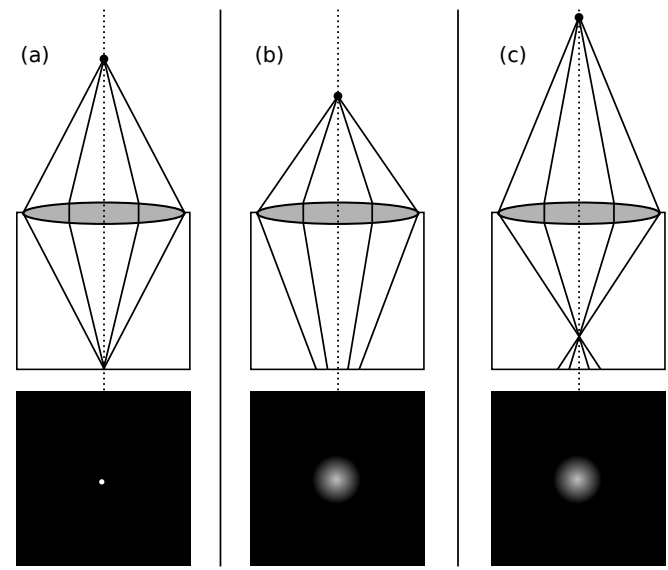

Figure 1: Illustration of single-lens stereo concept. Adapted from Adelson and Wang. ${ }^{14}$
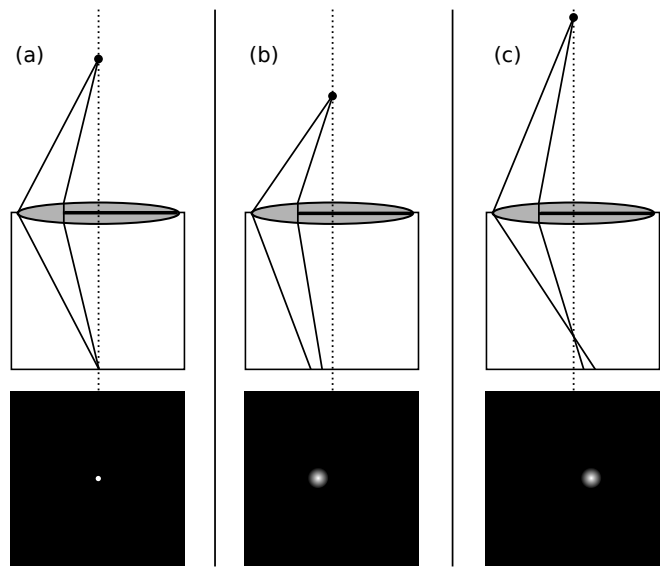

Figure 2: Illustration of imaging with an eccentric aperture. Adapted from Adelson and Wang. ${ }^{14}$
The plenoptic camera operates on a similar concept where a microlens array is used to encode the angular information of incident rays onto pixels found behind each microlens. This is illustrated in figure 3 with pinholes used in place of microlenses. In this case, a main lens is used to form an image on the array of pinholes with $3 \times 3$ pixels located behind each pinhole. As such, each pinhole represents a macropixel. When an object is perfectly focused on the center pinhole (fig. 3a), all of the rays converge at the pinhole illuminating all of the pixels found underneath that particular pinhole. When the object is moved closer (fig. 3b), however, a blurred spot is produced that spans several pinholes. As the angle of rays reaching the pinholes varies depending on the pinhole location, only certain pixels under each pinhole receive light whereas the others remain dark. This is illustrated by the pattern of pixels found beneath each pixel in the figure. Conversely, when the the object is moved further away, the pattern under each pinhole is different (fig. 3c). As such, by analyzing the distribution of
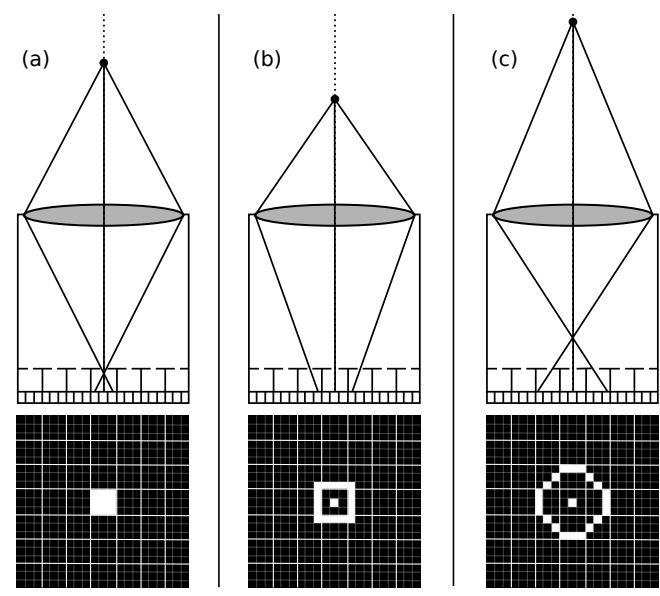

Figure 3: Illustration of plenoptic camera based on an array of pinhole cameras. 
light under each pinhole, the depth of the object can be determined. Replacing each pinhole with a microlens yields the same result, but greatly increases the amount of light collected by the sensor.

This concept was demonstrated by Adelson and Wang ${ }^{14}$ using a 500 x 500 pixel CCD camera with microlenses forming $5 \times 5$ pixel macropixels. As such, the spatial resolution of the resulting image was $100 \mathrm{x}$ 100 pixels with the 25 pixels under each macropixel used to record angular information about the image. This illustrates an inherent tradeoff associated with the plenoptic camera between spatial and angular resolution. Nonetheless, Adelson and Wang were able to use their prototype sensor to demonstrate the concept as well as for rangefinding where they produced qualitatively accurate depth maps for various scenes.

\section{B. Recent Developments}

Interest in plenoptic cameras has picked up recently due to the rapid increases in CCD resolution which allow both the spatial and angular resolution to be adequately sampled. In particular, we note the work of $\mathrm{Ng}$ et al. ${ }^{15}$ who developed a hand-held version of the camera using a commercially available 16 megapixel image sensor and a micro-lens array consisting of $296 \times 296$ microlenses. Their focus was on digital photography where the additional information made available with a plenoptic camera allows for the computation of synthetic photographs that allow for focusing of the camera or adjustment of the aperture after the image has been taken. Also demonstrated in their work was the ability to move the observer across the aperture of the camera, which produces changes in parallax. This is particularly useful in macro (close-up) imaging as is often used in the laboratory and wind tunnel environment. The number of views available is equal to the number of pixels behind each microlens. In their case, this corresponded to a total of 196 (14 x 14) different views of the same scene recorded on a single sensor. This will prove to be an important aspect when we consider the idea of tomographic reconstruction, where by comparison with traditional imaging, 196 viewpoints using separate cameras is not practical.

More recently, efforts have been underway by Levoy et al. ${ }^{16,18}$ to develop a lightfield microscope based on the plenoptic camera. The fundamental principle remains the same; however, their work focused on additional challenges associated with microscopic imaging. For one, wave optics and diffraction must be considered in a microscopic environment whereas geometrical optics is sufficient for macroscopic imaging. In addition, a typical microscope objective functions differently than a normal camera lens, producing orthographic rather than perspective views. Next, most objects in microscope images are partially transparent whereas the previous effort had focused on scenes with opaque objects. This last point is the most relevant to the proposed work where particle fields are also transparent. This feature allows for the use of 3-D reconstruction algorithms to render a volumetric image of the object. The choice of algorithm is the primary challenge in developing 3-D diagnostics using plenoptic imagery. In this paper, we focus on a tomographic reconstruction approach because the approach using refocusing and thresholding is limited by particle number density.

This paper begins first by describing the development of a simulator for generating synthetic images of particle fields. These simulated images are used as inputs for the reconstruction algorithms. The results of these algorithms will be used as inputs to a 3-D cross-correlation algorithm to extract a velocity field. This set of processing steps is then extended for use on preliminary experimental data acquired with a prototype based on a 16-megapixel camera.

\section{Camera Simulation}

The use of linear (Gaussian) optics is well established for geometrically tracing the path of light through space and various optical elements by the use of matrix methods from linear algebra. An important application of Gaussian optics is ray tracing in computer graphics. Briefly, ray tracing is a rendering technique in which a large number of light rays from a scene are used to form an image at arbitrary locations or viewpoints. Rays of light are initialized at the light source by specifying an initial position and direction. Any number of ray transfer matrices are then used to simulate optical elements and the propagation of light through free space. ${ }^{19}$ The intersection each ray makes with a sensor plane or designated viewpoint defines the generated image. Georgeiv and Intwala ${ }^{20}$ have shown that Gaussian optics can be extended to light field imaging as well, through an extension of basic linear optics known as affine optics. The simulation constructed here applies their work with affine optics to the concept of ray tracing, allowing simulated light field images of 3 -D particle fields to be generated. It should be noted that this generalized approach is necessary due to the complex optical configuration of the camera, which precludes the use of traditional synthetic PIV image 
generation schemes.

In Figure 4, the optical elements comprising the simulation are shown (not to scale), and the corresponding dimensional variables of the simulation are labeled. The origin of the optical axis is located at the sensor plane, with the z-axis oriented out of the camera through the center of the lens aperture, and the $\mathrm{x}$ - and $\mathrm{y}$-axes aligned with the sensor plane (the $\mathrm{x}$-axis is projected out of the page in this figure). The optical setup can be described using the thin lens equation; $s_{o}$ is the distance to the focal plane, and $s_{i}$ is the distance to the image plane of the main lens. When defined in this manner, the main lens of focal length $f_{m}$ acts to form a focused image at the image plane, exactly like a conventional camera. However, rather than placing the sensor at the image plane, a microlens array is inserted, with individual microlens focal lengths $f_{l}$ and diameters $p_{p}$. The image sensor is then placed at the focal plane of the microlens array.

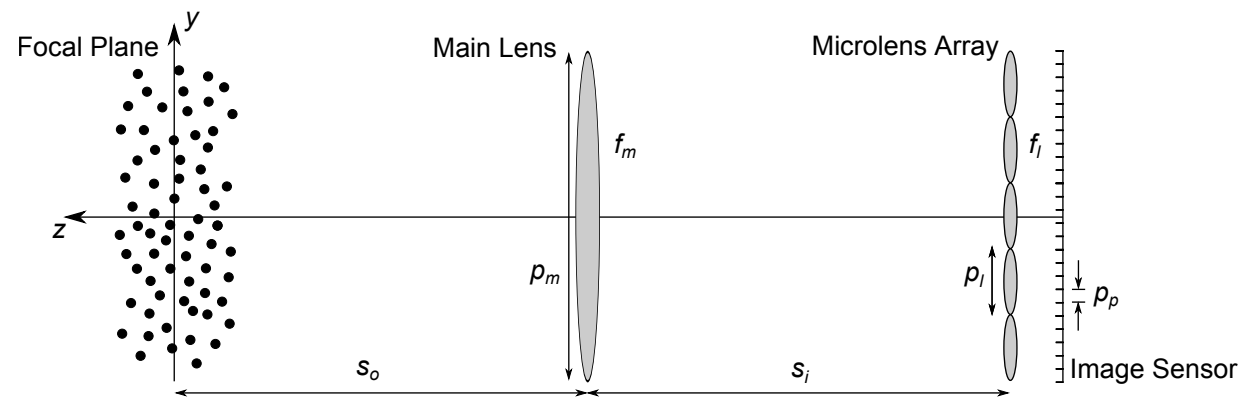

Figure 4: Schematic of Plenoptic Camera Optical Configuration.

All particles are approximated as point sources of rays. This simplification allows the particle field to be defined as simply the coordinates of each point within a specified volume with respect to the focal plane. This also allows the particles to be displaced by defined amounts to simulate particle motion between two images, as required by PIV. To constrain the design space so that the focus can be on algorithm development, all simulations use a $50 \mathrm{~mm}$ main lens focal length, magnification of 1 , microlens diameter of $125 \mu \mathrm{m}$, and microlens focal length of $500 \mu \mathrm{m}$. These constraints are set by the specifications of current commercially available microlens arrays. Also, the pixel size is $6.45 \mu \mathrm{m}$, selected in accordance with the specifications of the Kodak KAI-16000 image sensor.

\section{A. 1-D Simulations}

The 1-D simulator is used as a simple means to evaluate basic camera concepts without requiring a full image simulation, and is far easier to visualize. A detailed description of the simulator construction is given in Lynch. ${ }^{21}$ In figure 5a, the particle is placed at a distance $1 \mathrm{~mm}$ farther from the focal plane, resulting in a unique signal being generated. Conversely, in figure $5 \mathrm{~b}$, the particle is moved $1 \mathrm{~mm}$ closer, resulting in a completely different signal pattern. Multiple particles are able to be simulated, however these cases are not shown here since the visualization becomes difficult.

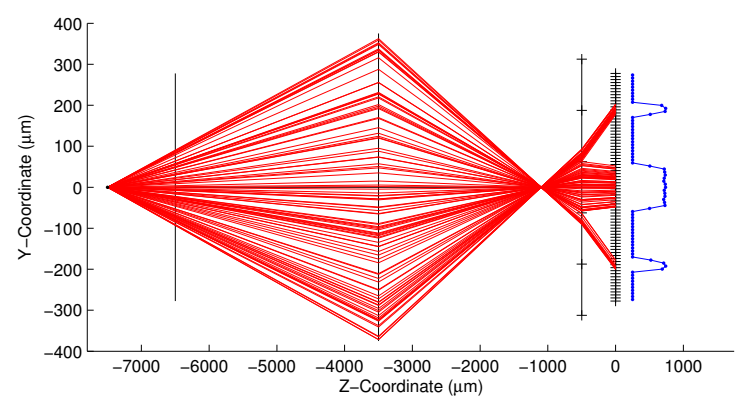

a) $d z=+1 \mathrm{~mm}$

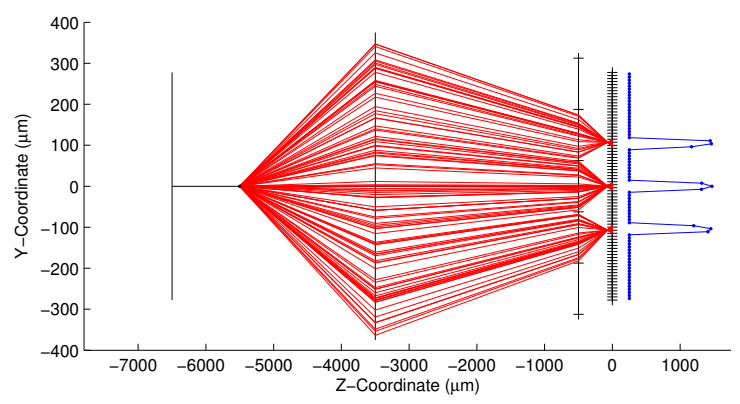

b) $d z=-1 \mathrm{~mm}$

Figure 5: 1-D Simulations at various particle depths. 1 out of every 100 rays shown. Integrated signal shown in blue. 
It should be noted that the simulator takes into account diffraction effects by randomizing the spatial coordinate of each light ray at the microlens plane and sensor plane by using a normally distributed random number generator, set in a manner that the standard deviation is equal to the diffraction-limited spot size. Through analysis it is seen that diffraction does not result in a substantial change in the simulator results. This is due to the large f-numbers used by the main lens and the microlenses. For the microlenses used in this study, the ratio of focal length to diameter leads to an f-number of 4 . As first explained in $\mathrm{Ng},{ }^{15}$ the effective f-number of the main lens must be equal to the microlens f-number to prevent microlens images from overlapping. For this reason, both the main lens and microlenses operate at a low f-number where diffraction effects are on the order of or smaller than the pixel size.

\section{B. 2-D Simulations}

The 1-D simulator has been extended to produce 2-D images of particles distributed within a volume. The ray-tracing procedure is identical to the 1-D simulator; however, the scale of the computation is orders of magnitude larger so the algorithms were ported and compiled in $\mathrm{C}$ for acceleration. The full description and source code listings of this program can be found in Lynch. ${ }^{21}$ The image size is set in accordance with the KAI-16000 image sensor to $4872 \times 3248$ pixels. A cropped portion of one of the generated images is shown in figure 6 . This image was generated using a particle volume ranging from $z=-10 \mathrm{~mm}$ to $+10 \mathrm{~mm}$ and a particle density of 0.001 particles per pixel (ppp). Upon a visual inspection of the image, particles that lie near the focal plane produce nearly circular images that stand out from the rest of the field. The remaining particle images are distributed across multiple microlenses and are difficult to distinguish visually.

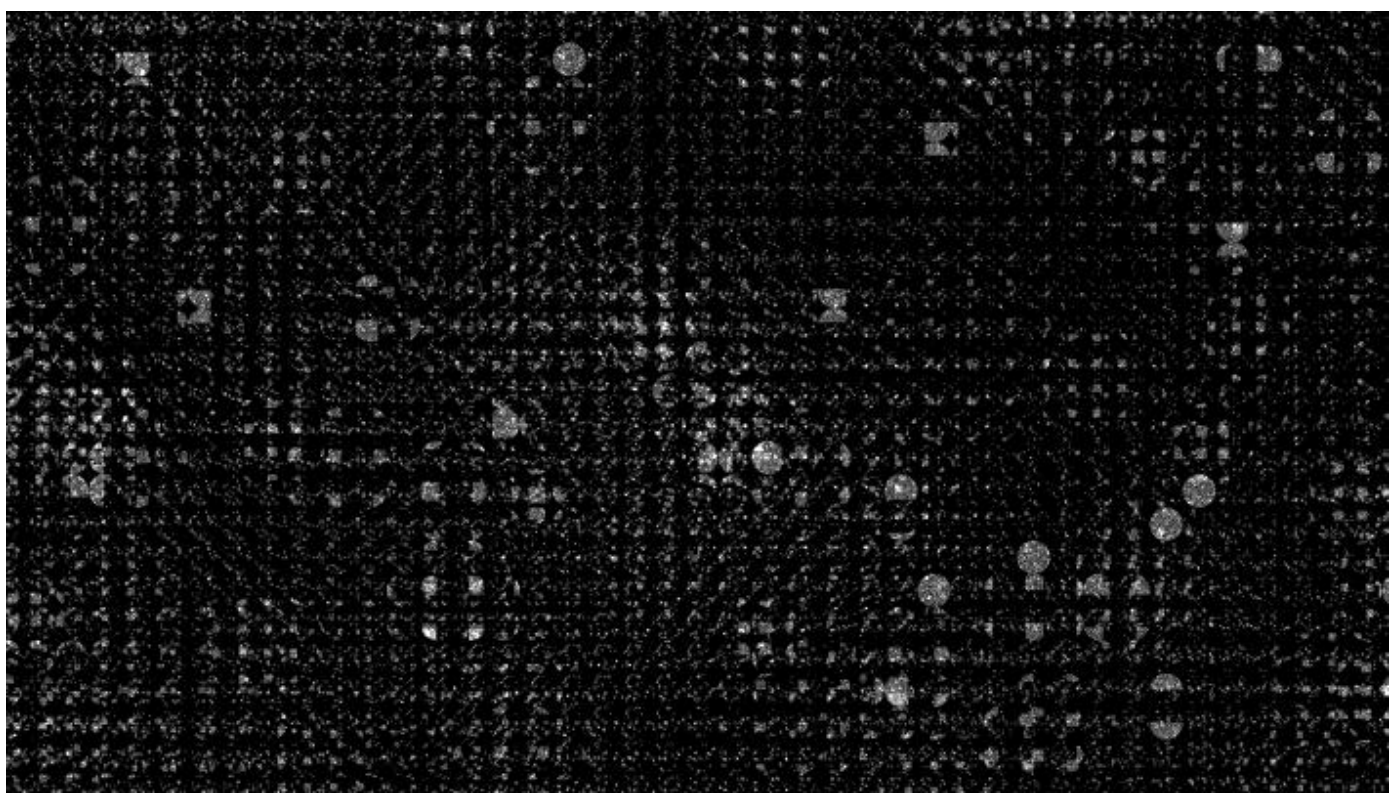

Figure 6: Example plenoptic image generated using the ray-tracing simulator.

\section{Image Processing}

As shown in figure 6, a raw plenoptic camera image, whether real or simulated, has a complex structure and is not amenable to direct analysis. In this section, the algorithms used to process the raw images are detailed.

\section{A. Geometrical Description}

Parameterizing the light field with two spatial coordinates and two angular coordinates, $L(x, y, \theta, \phi)$, is an intuitive way to describe light rays traveling through space. However, another approach can be used where the light rays are parameterized by their intersection with two planes, as described in $\mathrm{Ng} .{ }^{15} \mathrm{~A}$ ray in the light 
field is then described by four spatial coordinates: the position of intersection with the first plane located at microlens array $(x, y)$, and the position of intersection with the second plane at the main lens $(u, v)$. The first two coordinates are equivalent in both the angular and two-plane parameterization, and the last two coordinates are analogues to $(\theta, \phi)$ in the angular parameterization.

Since each microlens can be thought of as a miniature camera imaging the main lens, each pixel behind the microlens samples a unique part of the main lens as depicted in figure 7 where each rectangle represents a unique pixel. The non-plaid nature of the $x-u$ space comes about because each microlens images a slightly different and sometimes overlapping aperture due to their positions relative to the optical axis. While the parameterization may appear more complex due to this issue, the main advantage is its applicability to interpolation which serves as the basis for both the computational refocusing algorithm as well as the weighting function used for tomographic reconstruction.

\section{B. Computational Refocusing}

The concept behind light field rendering is to select a subset of rays from the complete 4-D light field to generate a conventional 2-D image. As mentioned previously, an advantage to using a two-plane parameterization is the ease of which it can be used as part of an interpolation algorithm suitable for refocusing. An illustration of the geometry used in the refocusing process is shown in figure 8 . The process of computational refocusing is based on a resampling of the light field inside the camera and extracting a proper slice of the resampled data which represents a subset of the light field corresponding to a specific depth.

To resample the light field a virtual image sensor $x^{\prime}$ is placed at a distance $s_{i}^{\prime}$ from the main lens plane, corresponding to a different object distance than the original sampling of the light field (therefore a different focal plane). This virtual light field, denoted $L_{F}^{\prime}$, can be written in terms of the sampled light field $L_{F}$ by a linear projection as shown in figure 8. Mathematically, the location of the projection of $x^{\prime}$ onto $x$ for a single $u$ value, denoted $x_{\text {find }}$, is given in equation 1 , where $\alpha=s_{i}^{\prime} / s_{i}$. For each $x^{\prime}$ location (location of a microlens on the virtual sensor plane), there will be a different $x_{\text {find }}$ value associated with each $u$. Thus the intensity at $x^{\prime}$ is the summation of the intensities of the $x_{\text {find }}$ locations of the sampled light field.

The virtual light field at a location $\left(x^{\prime}, y^{\prime}\right)$ expressed in
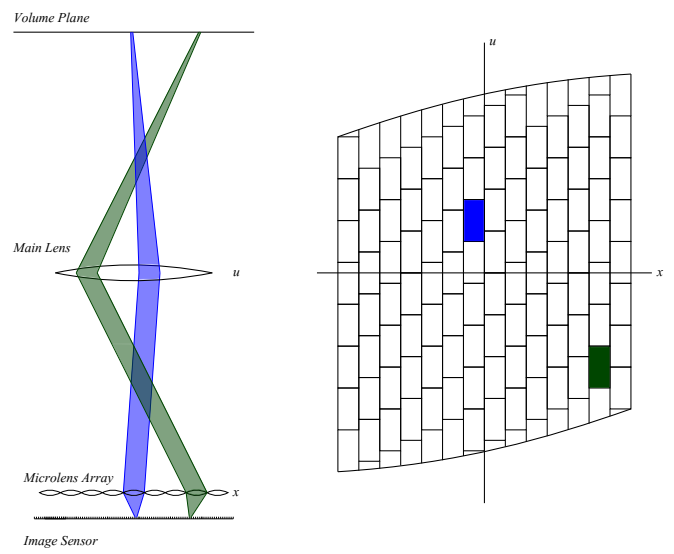

Figure 7: Illustration of two-plane paramaterization. Adapted from Ng. ${ }^{15}$

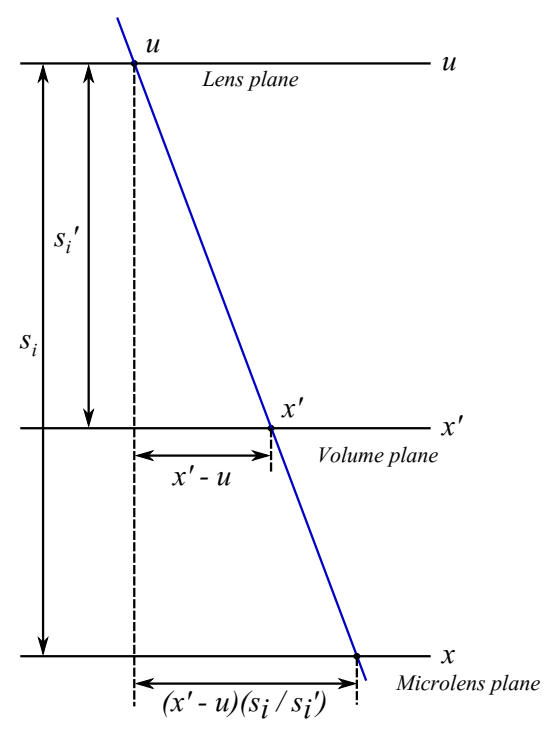

Figure 8: Schematic of two-plane interpolation, Adapted from Ng. ${ }^{15}$ terms of the sampled light field is described by equation 2 . Due to the nonuniformity in the $u$-plane associated with each microlens and that the projection may not necessarily be located at an exact pixel location, an interpolation scheme is needed to properly determine the contribution of each pixel. The interpolation is a $4 \mathrm{D}$ interpolation in the $(x, y, u, v)$ space. An example of the algorithm applied to actual image data is shown in figure 9. The original image is shown in figure 9b, with 9a and 9c representing the refocused images closer and further away from the camera, respectively.

From an implementation standpoint, the refocusing procedure requires calibrating the microlens array with respect to the sensor plane. The Gaussian nature of diffraction allows a three-point peak fitting procedure to be used to locate the positions of the microlenses to sub-pixel accuracy. The final 2-D image is formed by integrating the pixels falling within a designated radius of each microlens center. 


$$
\begin{gathered}
x_{\text {find }}=\left(x^{\prime}-u\right) \frac{s_{i}}{s_{i}^{\prime}}+u=u\left(1-\frac{1}{\alpha}\right)+\frac{x^{\prime}}{\alpha} \\
L_{F}^{\prime}\left(x^{\prime}, y^{\prime}, u, v\right)=L_{F}\left(u\left(1-\frac{1}{\alpha}\right)+\frac{x^{\prime}}{\alpha}, v\left(1-\frac{1}{\alpha}\right)+\frac{y^{\prime}}{\alpha}, u, v\right)
\end{gathered}
$$

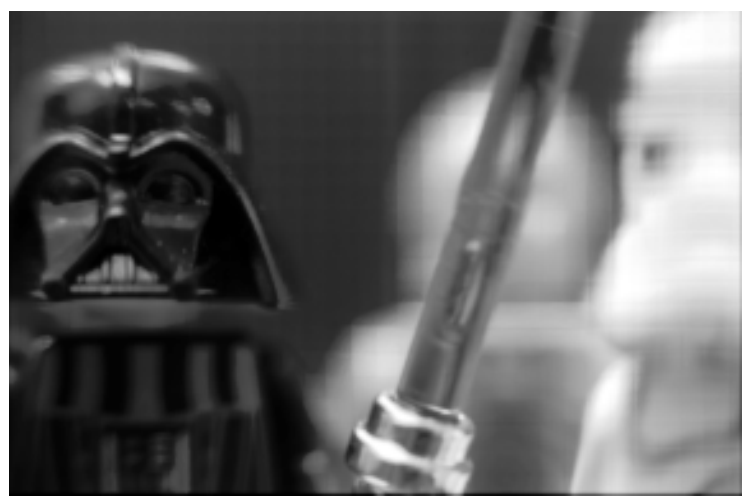

(a) Middle

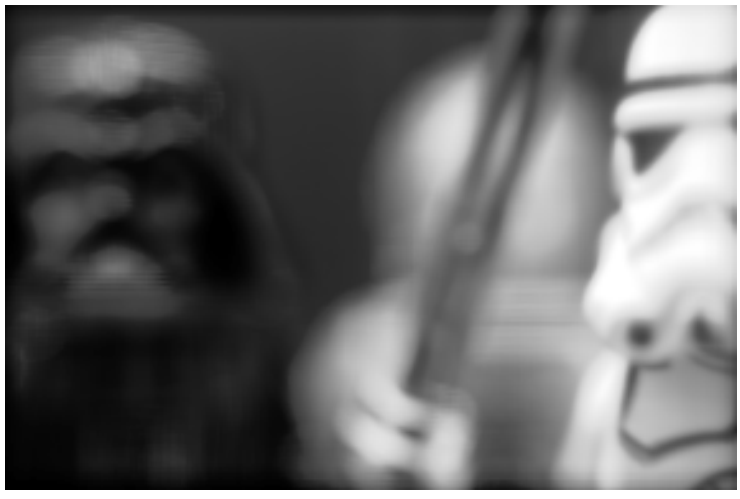

(b) Front

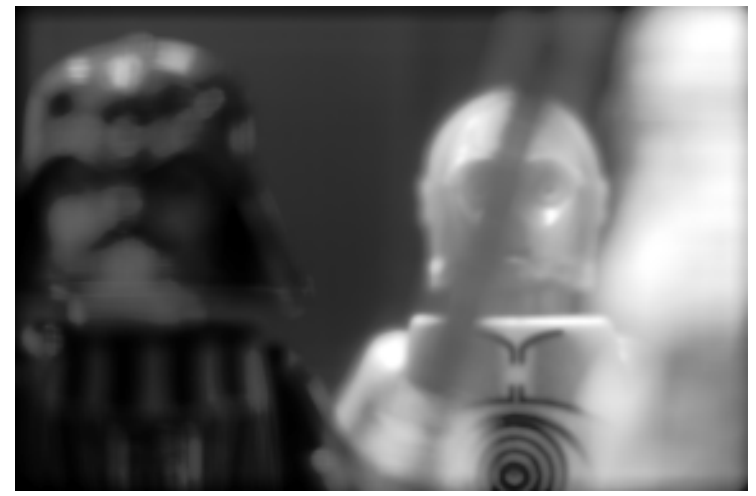

(c) Back

Figure 9: Examples of computationally refocused images using actual camera images.

\section{Perspective Views}

Perspective images are generated by taking a single pixel behind each microlens; in essence, the same $(u, v)$ for each $(x, y)$. In this manner, it is possible to show the angular range able to be measured by the camera. Also, the three-dimensional nature of the scene can be visualized by using multiple perspective views to show parallax between them. While not directly applicable to the analysis techniques developed in this work, perspective views may represent a capability which may find other utilities. Some sample images of this effect are shown in figure 10.

\section{Tomographic Reconstruction}

A more direct approach to analyzing the images is to use tomographic reconstruction procedures to generate a volume based on the large number of views available. For this purpose, an implementation of the multiplicative algebraic reconstruction technique (MART) is used, similar to that used in tomographic PIV. ${ }^{8}$ The primary challenge in implementing the MART algorithm with plenoptic camera data is the complex nature of the weighting function compared to traditional cameras.

The weighting function is calculated in a similar manner to how refocused images are generated. The relationship between a voxel and the imaging sensor is given through an interpolation of the light field. The voxel positions, located in object space, must first be transformed into image space using the thin-lens 


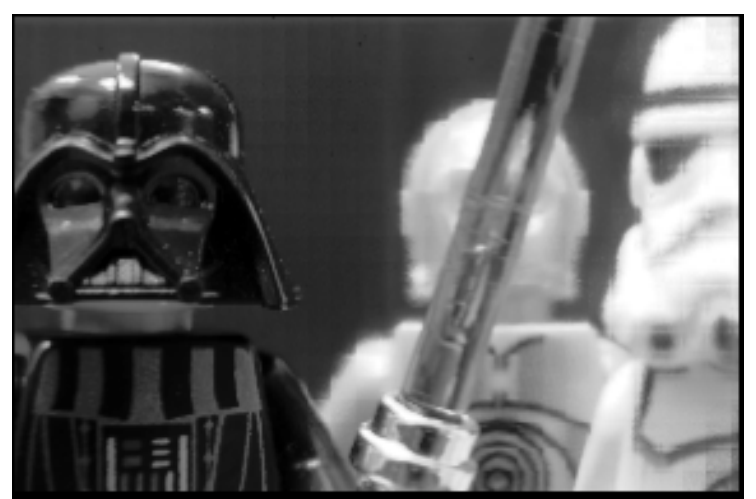

(a) Left

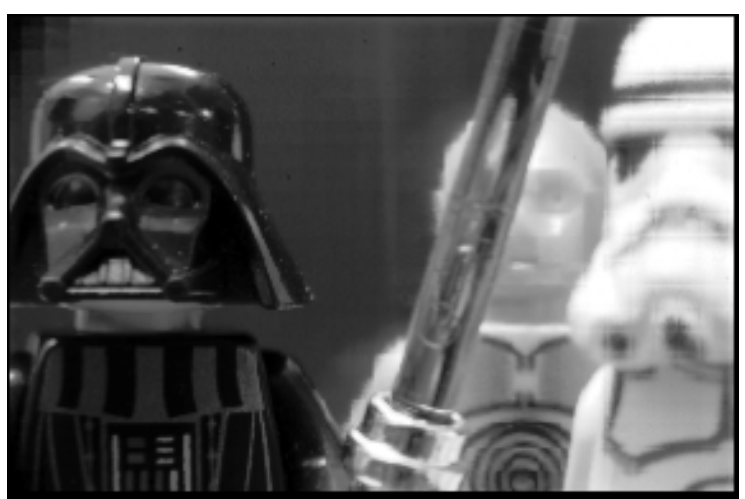

(b) Right

Figure 10: Perspective images

equation to yield the coordinate $x^{\prime}$ l. Since the voxels are in different locations and are of a different size than the microlenses, some modification must be made to the procedure presented for computational refocusing. One major adjustment is that it is assumed that the $(x, y, u, v)$ space is plaid, such that each microlens images an identical aperture. Since this is not how the optical system within the camera works, the light field image must be warped to generate a plaid array. This is performed by interpolating the predetermined $(u, v)$ values for each microlens onto a plaid $(u, v)$ distribution. The weighting function for each voxel is then given by the interpolation coefficents as shown in equation 3 .

$$
\begin{gathered}
N_{0000}=\left(x_{1}-x_{2}\right)\left(y_{1}-y_{2}\right)\left(u_{1}-u_{2}\right)\left(v_{1}-v_{2}\right) \\
N_{0001}=\left(x_{1}-x_{2}\right)\left(y_{1}-y_{2}\right)\left(u_{1}-u_{2}\right)\left(v_{2}-v_{0}\right) \\
\vdots \\
N_{1111}=\left(x_{2}-x_{0}\right)\left(y_{2}-y_{0}\right)\left(u_{2}-u_{0}\right)\left(v_{2}-v_{0}\right)
\end{gathered}
$$

where $\left(x_{2}, y_{2}, u_{2}, v_{2}\right)$ is the point of interest and the subscripts 0 and 1 refer to the neighboring points. $N_{0000}$ is the interpolation coefficient for the pixel $\left(x_{0}, y_{0}, u_{0}, v_{0}\right)$. The interpolation coefficent $N_{0000}$, is interpreted as the raw weight of the pixel $\left(x_{0}, y_{0}, u_{0}, v_{0}\right)$ for voxel $\left(x^{\prime}, y^{\prime}\right)$. The final step is to convert the raw weights to a normalized version by dividing by the total weight ensuring that the total contribution of a given voxel to the pixels is unity. For the $j$-th voxel, this results in equation 4 .

$$
w_{i, j}=w_{i, j} / \sum_{i} w_{i, j}
$$

The effect of the interpolation can be seen in Figure 11 where 3 voxels (a,b,c) of position $(7,7,15),(0,0,0)$, and $(-7,-7,-15)$ were interpolated onto the image sensor.

An additional concern, which arises from the thickness of the volumes is the need for super-sampling. If the voxels are located too close or too far from the camera, the normal $(u, v)$ can skip over microlenses as illustrated in figure 12. By super-sampling the $(u, v)$ plane, the distance between the rays decrease and no pixels are missed. This is further illustrated in figure 13 where a voxel identical to that in figure 11a is both normally sampled and super-sampled. The effect can be interpreted as missing rows and columns of pixels.

One of the substantial differences between the MART applied for tomographic PIV and the plenoptic camera is the number of pixels that image each voxel. In the current implementation, each voxel effects 4096 pixels, i.e., each of the 196 unique views are interpolated across 16 different pixels. Our current implementation is in its infancy and does not allow for the removal of redundant pixel values. This results in a weighting matrix that is $244 \mathrm{~Gb}$ for a grid resolution of $200 \times 200 \times 200$ voxels being imaged by a 16 megapixel sensor. These large file sizes also affect the time it takes to reconstruct a volume; a reconstruction of 5 iterations of MART for a volume of $200 \times 200 \times 200$ resolution takes approximately 12 hours. New methods are currently being explored to improve the algorithm in both of these areas. 


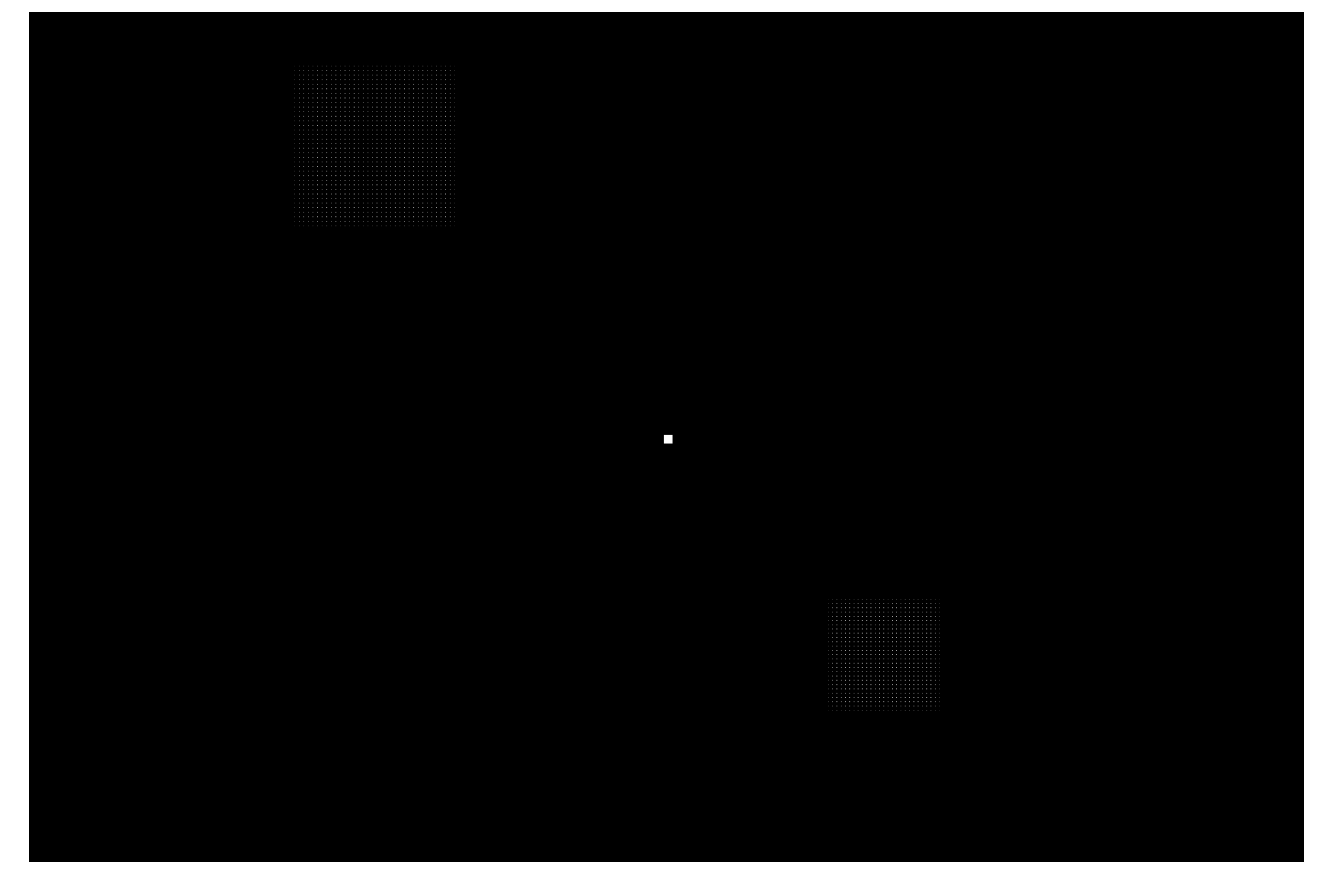

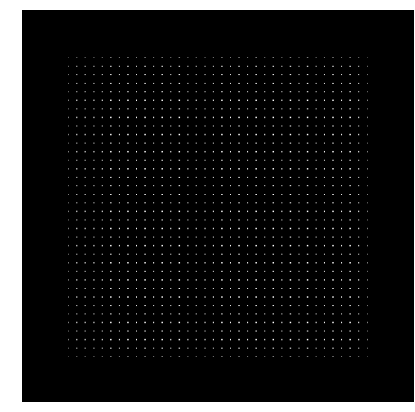

(a) $\alpha>1$

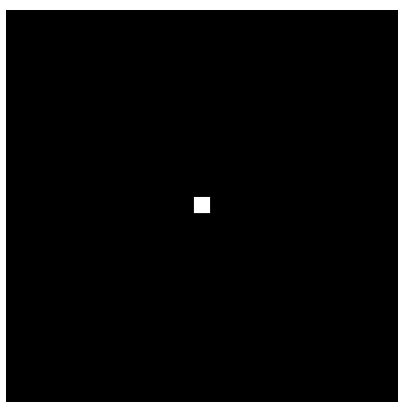

(b) $\alpha=1$

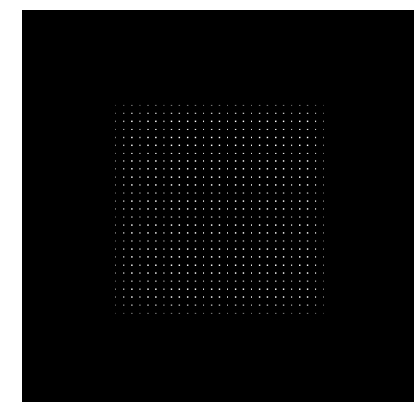

(c) $\alpha<1$

Figure 11: Interpolation of 3 voxels onto image sensor 


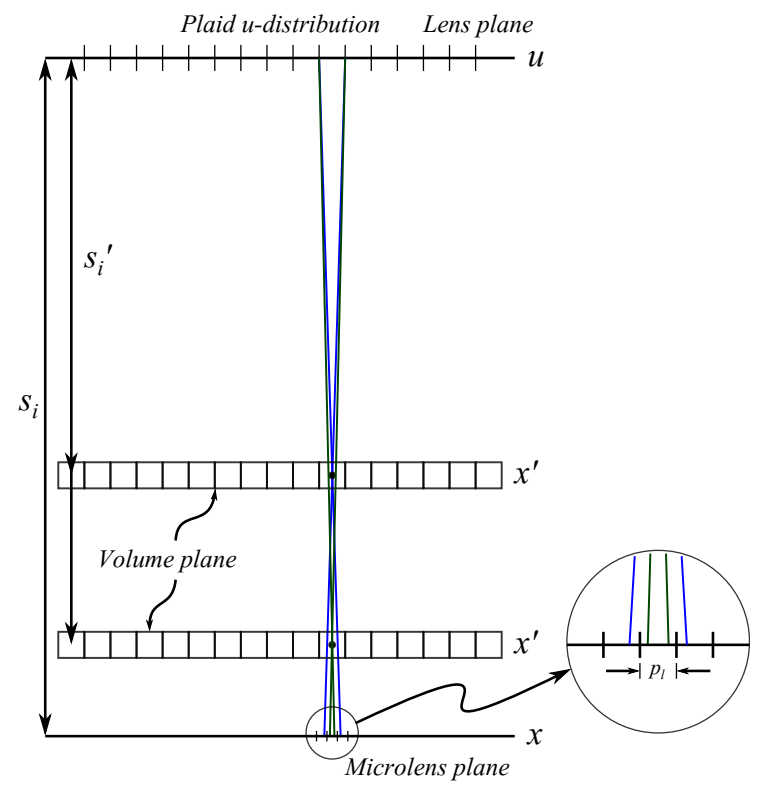

(a) Normal sampling of u-plane

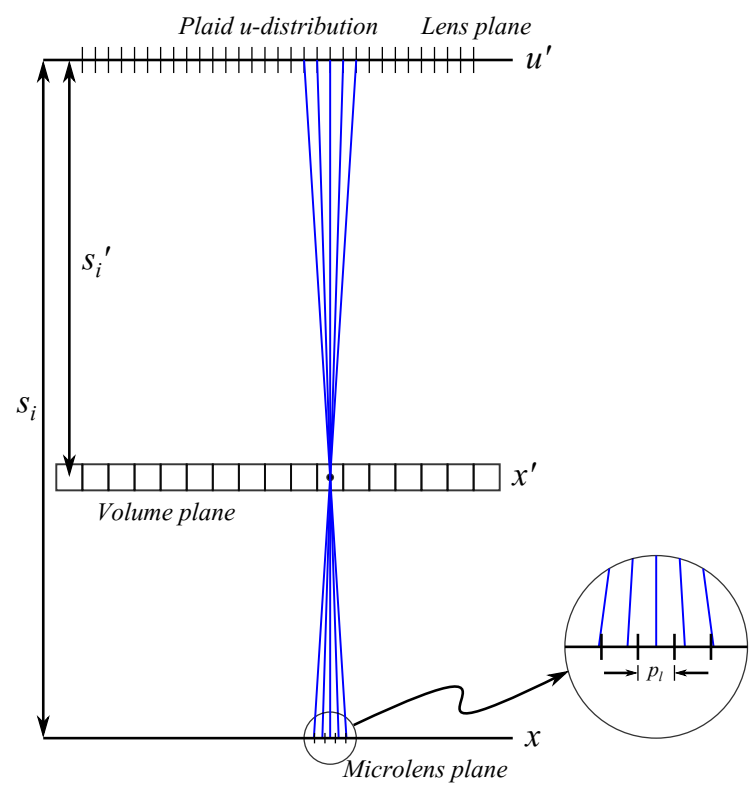

(b) Super sampling of u-plane

Figure 12: Illustration of super-sampling

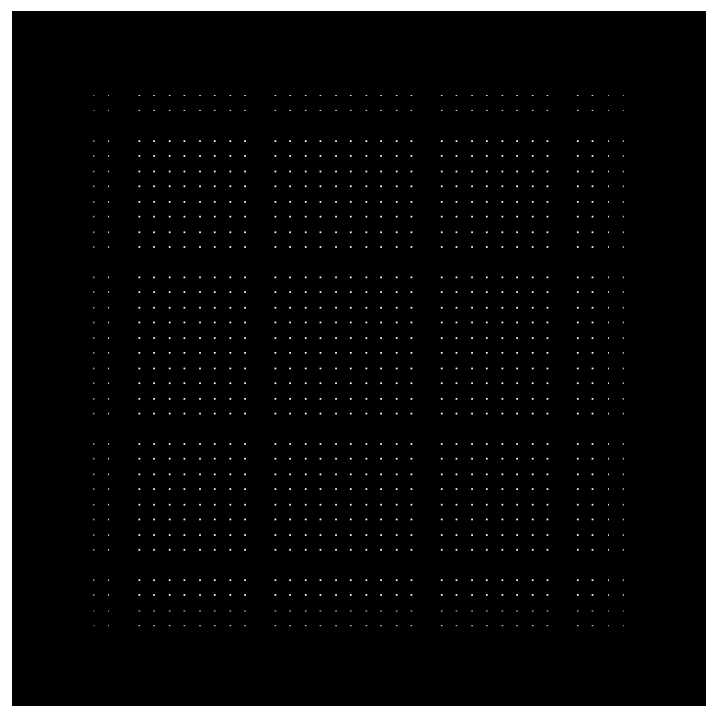

(a) Normal-sampling

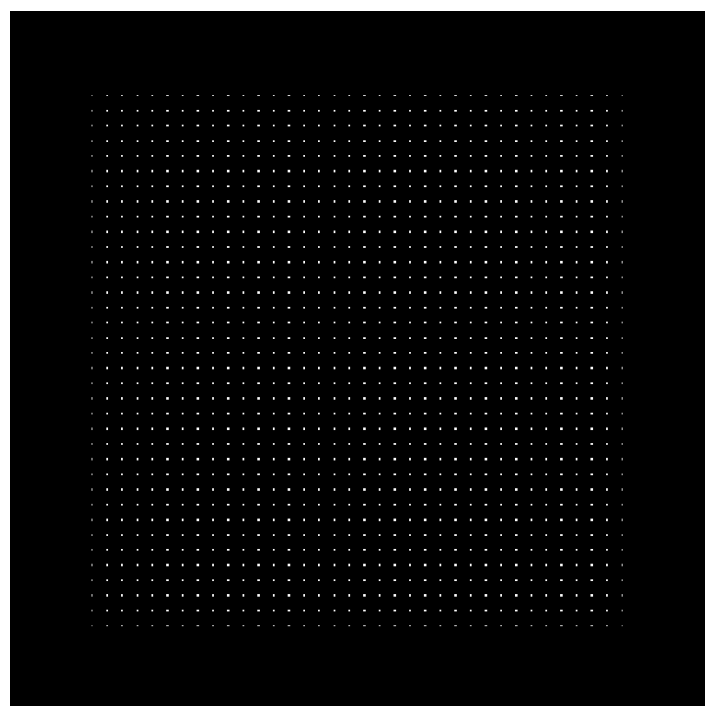

(b) Super-sampling

Figure 13: Effect of super-sampling 


\section{Simulated Results}

Testing of the simulation, rendering, and correlation algorithms is performed in this section by analyzing a case of a spherical vortex simulation. In the following results, the 3-D cross correlation is performed by an iterative volume deformation technique, first implemented by Scarano and Poelma. ${ }^{11}$

An example of a vector field evaluated from displacements based on an Oseen vortex line are shown in figures 14 and 15. The bulk motion is able to be captured, but there appears to be a distortion in the velocity field in the depth direction that must be addressed. The preliminary results reported here do not yet explore the accuracy of the technique in relation to the analytical displacement but will be the focus of a future study. However, these results indicate that the MART algorithm is capable of reconstructing a volume from plenoptic camera data and provides confidence in the technique.

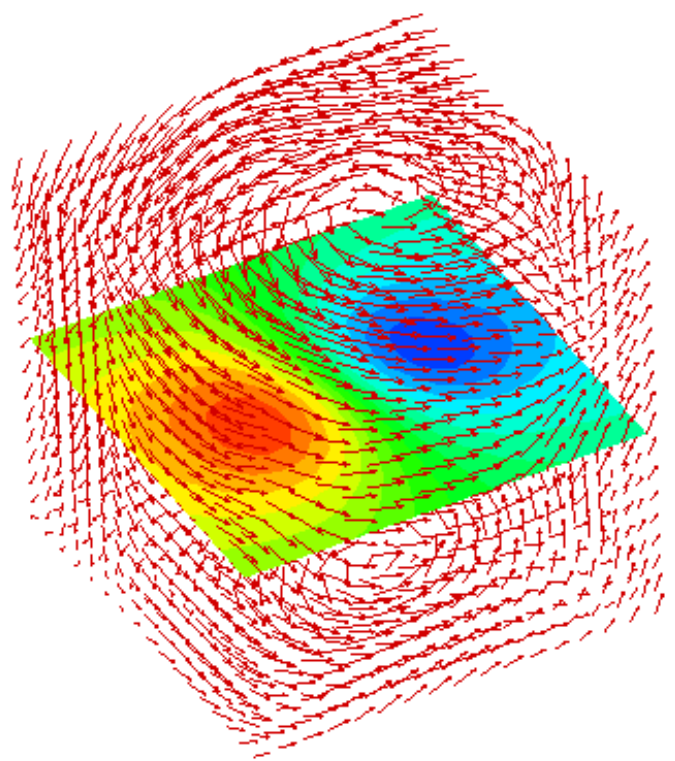

Figure 14: Velocity field measured with a vortical particle displacement.
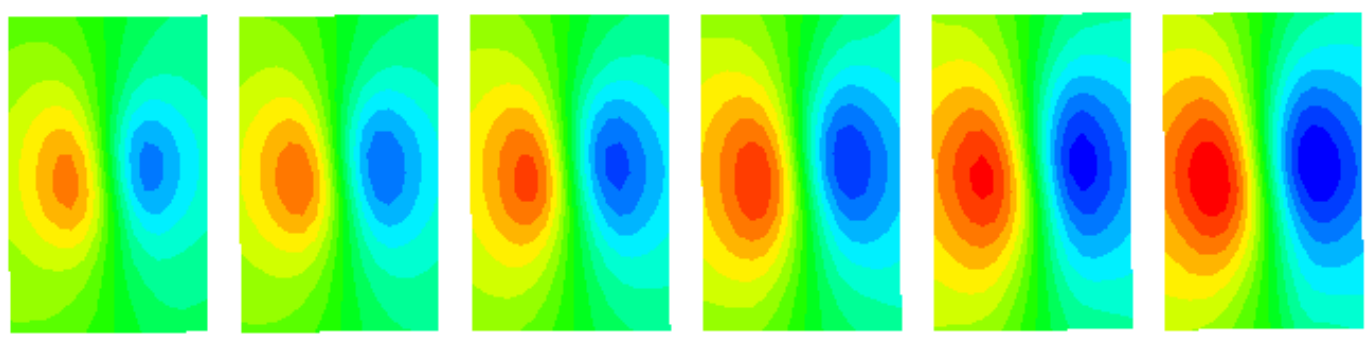

Figure 15: Velocity field measured with a vortical particle displacement.

\section{Prototype Construction}

To begin demonstrating the technique in an actual experiment, a prototype has been constructed using an Imperx Bobcat ICL-B4820. This camera is based on the Kodak KAI-16000 image sensor, the largest commercially available interline CCD. The choice of an interline CCD is motivated by the need to perform a double exposure similar to traditional PIV cameras. Figure 16 shows a photo of the camera without a

lens attached, and a quarter to provide scale. Immediately the compact design of the camera is evident, compared to the complex arrangements required for tomographic PIV or other methods. 


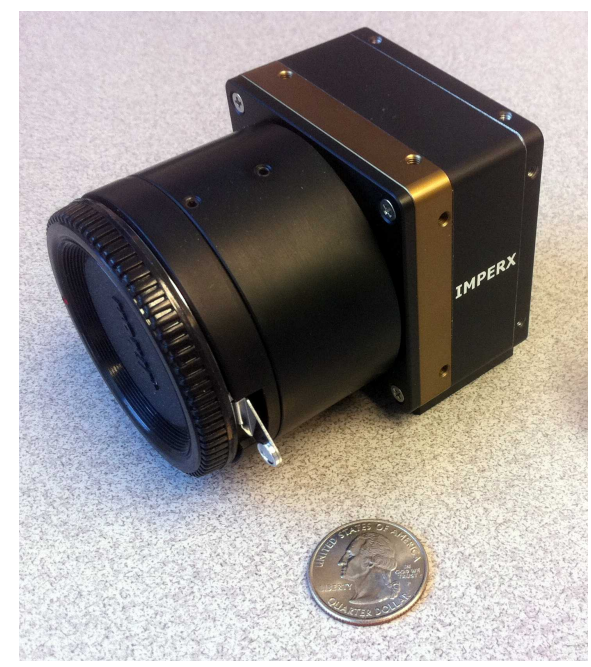

Figure 16: Imperx camera which is modified to become a plenoptic camera.

The microlens array is furnished by Adaptive Optics Associates, a subsidiary of Northrup Grumman. The challenge in constructing the prototype is fabricating a custom mounting device for the microlens array to position it accurately over the sensor. A custom mount has been designed by Light Capture, Inc., and has been manufactured in-house. An exploded view of these components and their relation to each other is shown in 17. The mount consists of a series of positioning screws to adjust the height of the microlens array above the sensor and to adjust the orientation of the array with respect to the sensor. To calibrate the and perform the adjustments, the camera is trained on a flat illuminated surface with the main lens removed. The design of the camera is such that the microlenses are exactly focused on the sensor. An iterative adjustment is then made so that the image captured by the camera is an array of sharp points. Note that the calibration only has to occur one time, a huge improvement over other techniques which require elaborate calibration for each experiment.

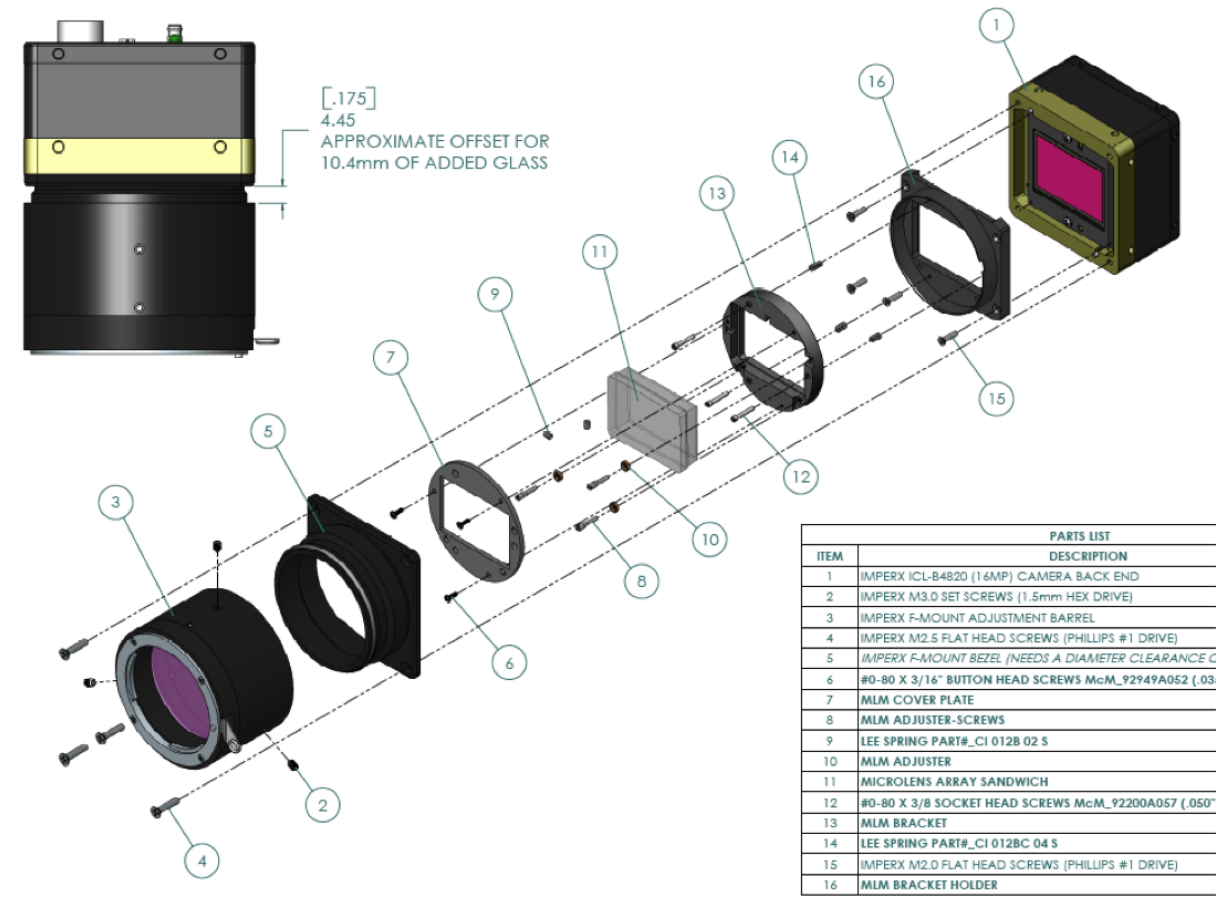

Figure 17: Exploded view of camera and microlens array mounting apparatus. 


\section{Conclusion}

A new technique for 3-D velocimetry based on a plenoptic camera has been detailed and demonstrated. A set of novel processing algorithms based on the MART tomographic reconstruction procedure have been described, which provide the ability to directly reconstruct a measurement volume from an acquired light field image. Following this procedure, cross-correlation algorithms are then used for extracting the velocity from the pair of volumes. This study has demonstrated this set of processing steps as viable for making 3-D velocity measurements, and has provided a foundation for further characterization of the performance of the technique and demonstration on experimental images.

\section{Acknowledgments}

This work has been supported through funding provided by the Air Force Office of Scientific Research. The authors would like to gratefully acknowledge Marc Levoy from Stanford University for permission to use a template for manufacturing our microlens array, and for a variety of helpful discussions. Additionally, the authors thank Stanley Reeves from Auburn University for continued discussions that have led us to consider the direct tomographic approach.

\section{References}

${ }^{1}$ Arroyo, M. P. and Greated, C. A., "Stereoscopic particle image velocimetry," Measurement Science and Technology, Vol. 2, 1991, pp. 1181-1186.

${ }^{2}$ Kahler, C. J. and Kompenhans, J., "Fundamentals of multiple plane stereo particle image velocimetry." Experiments in Fluids, Vol. 29, 2000, pp. S70-S77.

${ }^{3}$ Brucker, C. H., "Digital-particle-image-velocimetry (DPIV) in a scanning light-sheet: 3D starting flow around a short cylinder." Experiments in Fluids, Vol. 19, 1995, pp. 255-263.

${ }^{4}$ Zhang, W., Hain, R., and Kahler, C. J., "Scanning PIV investigation of the laminar separation bubble on a SD7003 airfoil," Experiments in Fluids, Vol. 45, 2008, pp. 725-743.

${ }^{5}$ Willert, C. E. and Gharib, M., "Three-dimensional particle imaging with a single camera," Experiments in Fluids, Vol. 12, 1992, pp. 353-358.

${ }^{6}$ Pereira, F., Gharib, M., Dabiri, D., and Madarress, D., "Defocusing digital particle image velocimetry: a 3-component 3-dimensional DPIV measurement technique. Application to bubbly flows." Experiments in Fluids, 2000, pp. S78-S84.

${ }^{7}$ Hinsch, K. D., "Holographic particle image velocimetry." Measurement Science and Technology, 2002.

${ }^{8}$ Elsinga, G. E., Scarano, F., Wieneke, B., and van Oudheusden, B. W., "Tomographic particle image velocimetry," Experiments in Fluids, 2006.

${ }^{9}$ Belden, J., Truscott, T. T., Axiak, M. C., and Techet, A. M., "Three-dimensional synthetic aperture particle image velocimetry," Measurement Science and Technology, Vol. 21, 2010, pp. 1-21.

${ }^{10}$ Schroder, A., Geisler, R., Elsinga, G. E., Scarano, F., and Dierksheide, U., "Investigation of a turbulent spot and a tripped turbulent boundary layer flow using time-resolved tomographic PIV," Experiments in Fluids, 2007.

${ }^{11}$ Scarano, F. and Poelma, C., "Three-dimensional vorticity patterns of cylinder wakes." Experiments in Fluids, 2009.

${ }^{12}$ Humble, R. A., Elsinga, G. E., Scarano, F., and van Oudheusden, B. W., "Three-dimensional instantaneous structure of a shock wave/turbulent boundary layer interaction," Journal of Fluid Mechanics, 2009.

${ }^{13}$ Violato, D., Moore, P., and Scarano, F., "Lagrangian and Eulerian pressure field evaluation of rod-airfoil fow from time-resolved tomographic PIV," Experiments in Fluids, Vol. 50, 2010, pp. 1057-1070.

${ }^{14}$ Adelson, E. H. and Wang, J. Y. A., "Single lens stereo with a plenoptic camera," IEEE Transactions on Pattern Analysis and Machine Intelligence, Vol. 14, 1992, pp. 99-106.

${ }^{15} \mathrm{Ng}$, R., Levoy, M., Bredif, M., Duval, G., Horowiz, M., and Hanrahan, P., "Light Field Photography with a Hand-held Plenoptic Camera," Tech. rep., Stanford, 2005 2006.

${ }^{16}$ Levoy, M., Ng, R., Adams, A., Footer, M., and Horowiz, M., "Light Field Microscopy," ACM Transactions on Graphics,

${ }^{17}$ Levoy, M., "Light Fields and Compuational Imaging," IEEE Computer, 2006, pp. 46-55.

${ }^{18}$ Levoy, M., Zhang, Z., and McDowall, I., "Recording and controlling the $4 \mathrm{D}$ light field in a microscope using microlens arrays," Journal of Microscopy, 2009.

${ }^{19}$ Gerrard, A. and Burch, J. M., Introduction to Matrix Methods in Optics, Wiley Interscience, 1975.

${ }^{20}$ Georgeiv, T. and Intwala, C., "Light Field Camera Design for Integral View Photography," Tech. rep., Adobe Systems, 2003.

${ }^{21}$ Lynch, K., Development of a 3-D Fluid Velocimetry Technique Based on Light Field Imaging, Master's thesis, Auburn University, 2011. 